Tohoku J. Exp. Med., 2005, 207, 217-222

\title{
Development of a Food-Frequency Questionnaire to Measure the Dietary Calcium Intake of Adult Japanese Women
}

\author{
Yuho Sato, Junko Tamaki, ${ }^{1}$ Fujiko Kitayama, ${ }^{2}$ Yukinori Kusaka, ${ }^{3}$ \\ Yumi Kodera, ${ }^{4}$ AkIKo Koutani ${ }^{5}$ and Masayuki IKI ${ }^{1}$ \\ Department of Nutrition, Tenshi College School of Nursing and Nutrition, \\ Sapporo, \\ ${ }^{1}$ Department of Public Health, Kinki University School of Medicine, Osaka, \\ ${ }^{2}$ Nutrient Control Room, Medical Service Section, University of Fukui Hospital, \\ Fukui, \\ ${ }^{3}$ Department of Environmental Health, University of Fukui Faculty of Medical \\ Sciences, Fukui, \\ ${ }^{4}$ Health Promotion Section, Fukui Health and Welfare Center, Fukui, \\ ${ }^{5}$ Nutritional Management Room, Fukui Prefectural Hospital, Fukui, Japan
}

Sato, Y., Tamaki, J., Kitayama, F., Kusaka, Y., Kodera, Y., Koutani, A. and Iki, M. Development of a Food-Frequency Questionnaire to Measure the Dietary Calcium Intake of Adult Japanese Women. Tohoku J. Exp. Med., 2005, 207 (3), 217-222 — Assessing calcium intake is necessary for epidemiological studies on osteoporosis. This study was aimed at developing a valid food frequency questionnaire (FFQ) to estimate the dietary calcium intake of Japanese people. The FFQ contains 26 items, representing common sources of calcium. The respondents, 74 women aged 15 - 79 years living in Fukui city, whose dietary calcium intake was estimated at $638 \pm 306$ (s.D.) $\mathrm{mg}$ based on a one-day weighted food record kept during the National Nutrition Survey of 1996, were asked to indicate their consumption frequency and usual portion size (large, medium, small) for each food item. Trained dieticians interviewed the subjects to confirm their answers to the FFQs. The mean calcium intake per day according to the FFQ $(655 \pm 222 \mathrm{mg})$ did not differ significantly from that according to the one-day food record. The coefficient of correlation between these methods was 0.512 , a statistically significant value. The calcium intake from dairy products showed a good correlation, $r=0.717$. The coefficient of correlation between the values estimated based on two FFQ filled out three days apart was 0.900 , showing good test-retest reliability. Therefore, this FFQ is valid and reliable in estimating dietary calcium intake for epidemiological studies. —— calcium; diet; food; nutrition; nutrition surveys

(C) 2005 Tohoku University Medical Press

Received May 30, 2005; revision accepted for publication August 30, 2005.

Correspondence: Yuho Sato, Kita-13, Higashi-3, Higashi-ku, Sapporo 065-0013, Japan.

e-mail: y-satou@tenshi.ac.jp 
Insufficient calcium intake is an established risk factor of osteoporosis. To prevent this disease, it is important to evaluate the calcium intake in populations at high risk for osteoporosis. However, there is as yet no ideal biological measure of dietary calcium intake for use in epidemiological surveys. The chemical analysis of a homogenate of the duplicate meals consumed by each subject gives an accurate estimate for calcium intake (Ueno et al. 2005), but is not feasible in large-scale epidemiological surveys. Several selfadministered diet assessment methods for Japanese people have been validated for various nutrients, including calcium (Sasaki et al. 1998; Tsugane et al. 2003), but they comprise more than 100 food items and require 40 to $60 \mathrm{~min}$ to complete. A simple food-frequency questionnaire (FFQ) for estimating the dietary calcium intake among Japanese people is necessary for largescale epidemiological studies, but has never been validated. Analogous FFQs have been used in Western countries (Cummings et al. 1987; Montomoli et al. 2002), but they cannot be used in Japan because the diets in those countries differ from the Japanese diet.

The dietary calcium intake in Japanese people, especially in Japanese women, is less than the minimum requirement of $600 \mathrm{mg} /$ day (Ministry of Health and Welfare 2004), which may have unfavorable effects on Japanese women's health. In this study, we aimed at developing a valid and simple FFQ to estimate the dietary calcium intake of the Japanese population living in Japan.

\section{Materials ANd Methods}

We chose women aged 15 - 79 years who lived in Fukui City and participated in the National Nutrition Survey of 1996 as eligible subjects. Two census tracts in Fukui City belonged to the group of 300 census tracts randomly selected throughout Japan for the National Nutrition Survey. These two census tracts included about 200 household members. $73.5 \%$ of the household members, including 77 women, participated in the National Nutrition Survey. Of them, 74 met the inclusion criteria of the present study and served as the subjects.

\section{The 26-item FFQ for calcium intake}

We constructed an FFQ listing 26 food and beverage items which are commonly ingested and also rich in calcium (Table 1). To complete the list of these items, we first selected dairy products, beans, fish, vegetables, and cereals as major food groups, in accordance with the report by the National Nutrition Survey in Japan (Ministry of Health and Welfare 1996). Second, we listed specific food items from each food group, which are consumed commonly by Japanese people and contain substantial amounts of calcium (Science and Technology Agency 1982). Additionally, we included other food items which were not in those food groups, but were calcium-rich nonetheless: eggs, sesame seeds and seaweed. The FFQ did not include calcium-fortified foods, soft drinks or calcium supplements.

Three serving sizes (large, medium and small) were listed, all relative to the medium portion size specified in the FFQ. The medium portion sizes for dairy products, "Natto," fish, and bread reflected the standard portion sizes commonly sold in the market. Those for soybean products, except "Natto," and eggs were derived from the Food Exchange Lists (Japan Diabetes Society 1996). For seaweed and potatoes, they were derived from the Japanese Nutrition Requirement (Health Promotion and Nutrition Division 1994). The relative weights for the large and small servings were calculated so as to yield the highest correlation coefficient between the total calcium intake estimated based on the FFQ and that estimated based on the one-day food record. Assuming that a medium-sized portion corresponds to $100 \%$ of its weight, a small portion was stipulated as corresponding to $80 \%$ and a large portion to $120 \%$ of the weight of the mediumsized portion, as will be discussed later. We would have determined the relative weights in advance, if there the data needed for the volume estimation of each item had been available. The calcium content for each item was derived from the Standard Tables of Food Composition, fourth revised edition (Science and Technology Agency 1982), or the Standard Tables of Commercial Elaboration Food, seventh revised edition (Kagawa 1995).

Our FFQ required each subject to indicate her usual portion size and her frequency of consumption for each item during the previous week. The amount of calcium consumed from each item during that week was calculated by multiplying the portion size by the frequency of consumption for that item. The total amount of calcium intake over a week was calculated as the sum of the amounts of calcium derived from all 26 items during that 
TABLE 1. Food items included in the FFQ, medium serving sizes, and calcium contents

\begin{tabular}{|c|c|c|c|}
\hline Food item & Food/Beverage name & Medium serving a $(\mathrm{g})$ & $\begin{array}{c}\text { Calcium content } \\
(\mathrm{mg})\end{array}$ \\
\hline 1 & Milk, drinkable yogurt & $200 \mathrm{ml}$ & 200 \\
\hline 2 & Yogurt & $100 \mathrm{ml}$ & 120 \\
\hline 3 & Skim milk & 16 & 180 \\
\hline 4 & Cheese & 29 & 180 \\
\hline 5 & Small fish eaten whole & $-c, d$ & 120 \\
\hline 6 & Fish products which include edible bone and skin & $--^{c, e}$ & 50 \\
\hline 7 & One slice of fish & 70 & 50 \\
\hline 8 & Canned fish products & 60 & 130 \\
\hline 9 & Eggs, coturnix quail eggs & 50 & 30 \\
\hline 10 & Tofu & 100 & 90 \\
\hline 11 & Deep-fried tofu, deep-fried tofu balls & 60 & 160 \\
\hline 12 & Natto & 40 & 35 \\
\hline 13 & Soybeans, green soybeans, soybean flour & $-\mathrm{c}, \mathrm{f}$ & 40 \\
\hline 14 & Green vegetables & $-c, g$ & $120 / 100 \mathrm{~g}$ \\
\hline 15 & Vegetables except greens & $-\mathrm{c}, \mathrm{g}$ & $25 / 100 \mathrm{~g}$ \\
\hline 16 & Seaweed & - c,h & 10 \\
\hline 17 & Sesame & 1 teaspoon & 25 \\
\hline 18 & Potatoes, sweet potatoes, taro & 50 & 6.5 \\
\hline 19 & Rice & 150 & 3 \\
\hline 20 & Bread & 60 & 22 \\
\hline 21 & Sweet rolls & 60 & 20 \\
\hline 22 & Hamburgers & 1 piece & 50 \\
\hline 23 & Udon noodles, buckwheat noodles, ramen noodles & 1 bowl & 18 \\
\hline 24 & Miso-soup & 150 & 10 \\
\hline 25 & Milk coffee, milk tea & 1 cup & 60 \\
\hline 26 & Ice-cream, shake & 120 & 160 \\
\hline
\end{tabular}

${ }^{a}$ Portion size, in grams unless otherwise stated, corresponding to the medium portion stated in the questionnaire; small is 0.8 times the medium amount, and large is 1.2 times the medium amount.

${ }^{\mathrm{b}}$ Calcium content per portion of food. Values derived from the Standard Tables of Food Composition in Japan, 4th revised edition, or the Standard Tables of Commercial Elaboration Food, 7th revised edition.

${ }^{c}$ As the item consists of several kinds of food, the serving size for each food is indicated in the FFQ.

${ }^{\mathrm{d}}$ Three salted and dried whole sardines, three semi-dried Atlantic capelins, or ten boiled and dried whole Japanese anchovies contain $120 \mathrm{mg}$ of calcium, respectively.

${ }^{\mathrm{e}}$ One portion of boiled and dried whitebait, dried Sakura shrimps, or simmered whole Japanese sand lance in soy sauce and sugar is two tablespoons, one teaspoon, or half a tablespoon, respectively.

${ }^{\mathrm{f}}$ One portion of soybeans, green soybeans, or soybean flour is $40 \mathrm{~g}$, $60 \mathrm{~g}$, or $20 \mathrm{~g}$, respectively.

${ }^{\mathrm{g}}$ The quantity for each vegetable, i.e., $100 \mathrm{~g}$, was indicated in the FFQ.

${ }^{\mathrm{h}}$ One portion of dried wakame seaweed, dried hijiki seaweed or dried kombu seaweed is $1 \mathrm{~g}$, respectively. 
week. The daily calcium intake was estimated by dividing the total amount by 7 .

\section{Reference method}

The one-day food records kept as part of the National Nutrition Survey of 1996 were used as the reference material in this study. Each one-day food record included the weights of all food items eaten during one day, measured with a dial scale. In the reference study, dieticians explained to the subjects in advance how to weigh each food item and record the data. The nutrition values were derived from the Standard Tables of Food Composition in Japan, fourth revised edition (Science and Technology Agency 1982).

The subjects were asked to fill out the food record for one day in November, 1996; the condition was that it should not be a Sunday or a holiday. The FFQ was to be filled out on a weekday, within 7 days of the completion of the one-day food record (i.e., if a subject had completed her one-day food record on November 15th, 1996, she was asked to pick a day for filling out the FFQ between November 16th and November 22nd). The dieticians interviewed the subjects to confirm their answers to the FFQ and the listings in their food records.

\section{Test-retest reliability study}

To evaluate the consistency of the estimated calcium intake values obtained from repeated completions of the FFQ, a test-retest reliability study was preformed with 14 women aged 25 to 78 years (mean age: 45.0 years) living in Osaka prefecture. Each subject was asked to fill out one FFQ every other day, and three FFQs were filled out in total. Each time they filled out an FFQ, they were required to include information for the previous 7 days. Then, the estimated calcium intakes obtained from the repeated FFQs for the same subject were compared.

\section{Statistical analysis}

The Shapiro-Wilk test was performed to test for normal distribution of the estimated calcium intakes. Pearson correlation analysis was performed to compare the estimated values given by the two methods, to determine the relative weights of the large and small serving sizes, and to compare the reproducibility of the estimated values. The paired $t$-test was used to compare the means of the estimated values given by the two methods and by the reproducibility test. To assess the consistency of the results from the repeated FFQs, the within-person coefficient of variation (square root of within-person variance divided by the mean calcium intake) was calculated using a formula (Willett 1998a; Sempos et al. 1985). The analyses were performed using SAS version 6.08 for Windows (SAS Institute, Cary, NC, USA).

Informed consent was obtained from the participants, and the survey was performed under the institutional guidelines.

\section{Results}

The 74 women who completed both the FFQ and the one-day food record had a mean age of 47.1 years. The mean calcium intake ( \pm S.D.) obtained from the one-day food records was 655 $\pm 222 \mathrm{mg}$ /day (range: $109-1,560 \mathrm{mg}$ ), and the mean calcium intake obtained from the FFQ was $638 \pm 306 \mathrm{mg} /$ day (range: 249 - 1,262 mg). Both methods yielded normally distributed calcium intakes, and the mean values were not significantly different between the two methods $(p>0.05$ by paired $t$-test). The coefficient of correlation between the calcium intakes obtained with the two methods was $0.512(p<0.001)$.

The correlation coefficient was 0.544 when only the data for women aged 30 to 69 years were included. The correlation coefficients for the calcium intakes from dairy products, miso (soybean paste), cereals and seafood were $0.717,0.543$, 0.507 and 0.419 , respectively.

We requested each of the 14 subjects to fill out an FFQ three times a week, as a test-retest reliability study. The mean of 42 estimated values was $609 \mathrm{mg}$. The Pearson coefficients of correlation between the estimated values obtained from the first and second FFQ, the second and third, and the first and third were 0.964, 0.978 and 0.900, respectively, and the results were statistically significant $(p<0.001)$. The mean values given by the first, second and third FFQ were $641 \mathrm{mg}, 603$ $\mathrm{mg}$, and $584 \mathrm{mg}$, respectively. There were no significant differences between any two values using the paired $t$-test. The within-person coefficient of variation for the total calcium intake was $9.8 \%$.

\section{Discussion}

We developed an FFQ to estimate the calcium intake in Japanese people, especially women. 
The daily calcium intake estimated based on our FFQ showed a significant correlation with the daily calcium intake calculated based on the oneday food records kept as part of the National Nutrition Survey of 1996, a well-organized survey with standardized techniques. The correlation coefficient of 0.51 obtained in the present study is within the range generally obtained in validation studies (Willett 1998b), and similar to or higher than those published in previous reports (Sasaki et al. 1998; Tsugane et al. 2003), which demonstrates the validity of our FFQ. The test-retest reliability obtained in this study indicates this FFQ is reliable, compared with a similar previous reliability study where the correlation coefficient was 0.73 (Xu et al. 2000).

The coefficient of correlation between the calcium intakes estimated by the two methods increased when we included only the data for women aged 30 - 69 years, because most of them prepared the food in their respective households, and thus knew more about the food ingredients, the amounts of each ingredient, the cooking methods, etc. It is particularly important that the FFQ was valid for women in that age group, because they are the population most exposed to osteoporosis and are the primary target of osteoporosis prevention or treatment.

The calcium intakes for individual food items showed high correlations between the two methods, especially for dairy products $(r=0.717$ ) and miso (soybean paste, $r=0.543$ ). Dairy products are the predominant source of dietary calcium for all age groups in Japan (Ministry of Health and Welfare 2004). The bean food group is the third most frequently consumed source of dietary calcium in Japan (Ministry of Health and Welfare 2004). Additionally, as those food items are regularly included in the normal Japanese diet, the one-day intake should reflect the intake during a week or even longer. Taken together, these data support the validity of our FFQ as a method of estimating the dietary calcium provided by the Japanese diet.

As mentioned earlier, calcium-fortified foods and calcium supplements were not included in this study, even though such foods and supple- ments are sometimes used by Japanese people. There are two main reasons not to include calcium-fortified foods and calcium supplements in this study. First, the one-day food records used as the standard in this study do not refer to the intake of calcium supplements. Second, we thought that calcium-fortified foods or supplements cannot be included in an FFQ, because of the sheer variety of calcium-fortified foods and supplements commercially available.

This study has several potential weaknesses. One of these is that we based our conclusions on food records which were kept only for one day. However, our survey was performed in November (autumn in Japan) when there is relatively little seasonal variation in the Japanese diet. Thus, we believe that the data can be safely generalized to represent the Japanese diet.

In conclusion, our FFQ appears to be a valid and reliable method of estimating the dietary calcium intake of Japanese women belonging to a wide range of age groups. We conclude that this simple FFQ can be fruitfully used in epidemiological studies due to its validity, reliability and applicability to large-scale populations.

\section{Acknowledgments}

We would like to thank the personnel at the public health center of Fukui Prefecture for their assistance.

This FFQ, written in Japanese, can be downloaded from the homepage of the Department of Public Health, Kinki University School of Medicine. (http://www.med.kindai.ac.jp/pubheal/)

\section{References}

Cummings, S.R., Block, G., McHenry, K. \& Baron, R.B. (1987) Evaluation of two food frequency methods of measuring dietary calcium intake. Am. J. Epidemiol., 126, 796-802.

Health Promotion and Nutrition Division, Health Service Bureau, Ministry of Health and Welfare (1994) Recommended Dietary Allowances for the Japanese, the fifth revision. Daiichi Shuppan, Tokyo. (in Japanese)

Japan Diabetes Society (1996) Food Exchange Lists for person with diabetes, 5th ed., Japan Association for Diabetes Care and Education, Tokyo. (in Japanese)

Kagawa, Y. (1995) The Standard Tables of Commercial Elaboration Food in Japan, 7th ed., Kagawa Nutrition University Publishing Division, Tokyo. (in Japanese)

Ministry of Health and Welfare (1996) The National Nutrition Survey in Japan, 1994. Daiichi Shuppan, Tokyo. (in Japanese) 
Ministry of Health and Welfare (2004) The National Nutrition Survey in Japan, 2002. Daiichi Shuppan, Tokyo. (in Japanese)

Montomoli, M., Gonnelli, S., Giacchi, M., Mattei, R., Cuda, C., Rossi, S. \& Gennari, C. (2002) Validation of a food frequency questionnaire for nutritional calcium intake assessment in Italian women. Eur. J. Clin. Nutr., 56, 21-30.

Sasaki, S., Yanagibori, R. \& Amano, K. (1998) Self-administered diet history questionnaire developed for health education: a relative validation of the test-version by comparison with 3-day diet record in women. J. Epidemiol., 8, 203-215.

Sempos, C.T., Johnson, N.E., Smith, E.L. \& Gilligan, C. (1985) Effects of intraindividual and interindividual variation in repeated dietary records. Am. J. Epidemiol., 121, 120-130.

Science and Technology Agency (1982) Standard Tables of Food Composition in Japan, 4th ed., Printing Bureau, Ministry of Finance, Tokyo. (in Japanese)

Tsugane, S., Kobayashi, M., Sasaki, S. \& JPHC (2003) Validity of the self-administered food frequency questionnaire used in the 5-year follow-up survey of the JPHC Study Cohort I: comparison with dietary records for main nutrients. J. Epidemiol., 13, Suppl. 1, S51- S56.

Ueno, K., Nakamura, K., Nishiwaki, T., Saito, T., Okuda, Y. \& Yamamoto, M. (2005) Intakes of calcium and other nutrients related to bone health in Japanese female college students: A study using the duplicate portion sampling method. Tohoku J. Exp. Med., 206, 319-326.

Willett, W. (1998a) Nature of Variation in Diet. Nutritional Epidemiology Second version. Oxford University Press, Oxford. pp. 33-49.

Willett, W. (1998b) Reproducibility and Validity of FoodFrequency Questionnaires. Nutritional Epidemiology Second version. Oxford University Press, Oxford. pp. 101-147.

Xu, L., Porteous, J.E., Phillips, M.R. \& Zheng, S. (2000) Development and validation of a calcium intake questionnaire for postmenopausal women in China. Ann. Epidemiol., 10, 169-175. 